\section{Deleterious mutation and genetic recombination}

SIR-There are two theories of the evolution and maintenance of genetic recombination that invoke deleterious mutation: the mutation-load theory of Kondrashov', and Muller's ratchet ${ }^{2}$. Because of the ubiquity of deleterious mutation, these theories may deserve the serious attention of evolutionists interested in recombination. So it is important to know whether the rejection of these theories by Burt and Bell ${ }^{3}$ is justified.

If deleterious mutations combine synergistically in their effects on organism fitness (that is, the marginal fitness decrement caused by the $n+1$ th deleterious mutation is greater than that caused by the $n$ th) then a population with recombination will enjoy a lower mutation load than a population without recombination ${ }^{4}$. There is evidence that deleterious mutations do interact this way ${ }^{5}$. Therefore, as Kondrashov ${ }^{6}$ observed, deleterious mutation may underpin a powerful selective force favouring the evolution and maintenance of intrachromosomal recombination. But further analysis by Kondrashov' suggests that this force will be weak if the number of chromosomes is greater than one. This latter view was recently reiterated by Burt and Bell ${ }^{3}$ to justify their rejection of Kondrashov's theory. Kondrashov's analysis, however, is seriously misleading.

To understand why, consider the following. It is intuitively clear that alleles for recombination will be especially favoured only if they are statistically associated with the beneficial consequences of their action. For this reason, it is now common (for example, ref. 7) when modelling the evolution of recombination to suppose, quite reasonably ${ }^{8}$, that the allele increasing recombination is recessive and on the same chromosome where it is exerting its analysed effects. Hence the dominant alleles suppressing recombination are found on non-recombinant chromosomes and so are denied any benefits arising from the action of the recessive allele. Of course, a recombination gene may cause recombination on other chromosomes, but this fact may be ignored by modellers as the gene is unlinked to this consequence of its actions.

But in his analysis of selection at a recombination locus, Kondrashov considers only the selection arising from the effects of the locus on chromosomes other than its own. So it is only natural that he observed that selection for recombination is very weak. It is remarkable that he observed selection for recombination at all. What is more relevant to the evolution of recombination is the selection that arises from the effects of a recombination gene on its own chromosome. Much of Kondrashov's analysis of evolution in a one-chromosome genome can logically be interpreted as addressing this question he concludes that a recessive allele causing free recombination always goes to fixation. He does not analyse the strength of selection leading to this outcome, and the qualitative considerations presented above suggest the force will be much stronger than that arising from the effects of the recombination gene on other chromosomes. So Kondrashov's theory cannot be excluded as a relevant theory to explain intrachromosomal recombination in species with many chromosomes.

The Muller's ratchet theory of recombination observes that, in a finite population of non-recombining chromosomes, the class of chromosomes with the fewest deleterious mutations may be lost by chance, and that this loss will be irrevocable. Successive losses result in a steady deterioration of the population. A population with intrachromosomal recombination, on the other hand, can restore the optimal class through recombination. In the same simulation study that led him to doubt the power of his mutation-load theory, Kondrashov observed the rapid operation of Muller's ratchet in nonrecombining chromosomes.

Burt and Bell also consider Muller's ratchet to be unworthy of serious consideration as a theory of recombination although, as Charlesworth ${ }^{9}$ points out, it has obvious potential as an explanation of their observation ${ }^{10}$ of a positive correlation in mammals between age at maturity and 'excess chiasma number' (total chiasma number minus haploid chromosome number). Their rejection is based on Bell's previous conclusion" that Muller's ratchet is not a good explanatin of the patterns of sexual and asexual reproduction in nature. This is not the place to question the adequacy of this conclusion or the understanding of Muller's ratchet. It suffices to observe that the study of Burt and Bell is relevant to the question of what forces influence intrachromosomal recombination levels in sexual species, which is a different question from what forces maintain sex.

Red Queen theories of recombination are enjoying a vogue, for good reasons. It would be unfortunate if deleterious mutation theories were ignored for poor reasons.

Department of Zoology,

South Parks Road, Oxford OX1 3PS, UK

1. Kondrashov, A.S. Genet. Res, 44, 199-217 (1984).

Maynard Smith, J. The Evolution of Sex (Cambridge University Press, 1976).

Burt, A. \& Bell, G. Nature 330, 118 (1987)

4. Crow, J.F. in Mathematical Topics in Population Genetics (ed. Kojima. K.I.) 128-148 (Springer. Berlin, 1970).

Mukai, T. Genetics 61, 149-176 (1969)

Kondrashov, A.S. Genet. Res. 40, 325-332 (1982)

Bell, G. \& Maynard Smith, J. Nature 328, 66-68 (1987)

8. Fincham, J.R.S. et al. Fungal Genetics (Blackwell, Oxford, 1979)

9. Charlesworth, B. Nature 330, 116-117 (1987)

10. Burt, A. \& Bell, G. Nature 326, 803-805 (1987)

11. Bell, G. The Masterpiece of Nature: the Evolution and Genetics of Sexuality (Croom Helm, London, 1982).

\section{Particle distinguishability}

SiR-As discussed by John Maddox ${ }^{1}$, Costantini ${ }^{2}$ and Tersoff and Bayer ${ }^{3}$ feel the need to modify the traditional route to the three forms of statistics. Both papers reject the assumption of a priori probabilities, but for different reasons.

Costantini claims that the energy constraint in Maxwell-Boltzmann statistics destroys the equal a priori argument for the cells, while Tersoff and Bayer use it as a deus ex machina to go from classical to quantum statistics. Although Costantini is right in observing that the energy constraint does destroy the equal a priori probability argument, he cannot claim that the maximum of the multinomial distribution $P\left\{n_{i}\right\}$ $=\left\{N ! / n_{1} ! \ldots n_{m} !\right\} p_{1}^{n_{1}} \ldots p_{m}^{n_{m}}$ gives the Maxwell-Boltzmann distribution for the $n_{i}$ under particle and energy constraints because the a priori probabilities $p_{i}$ are unknown. Boltzmann avoided this by treating the product of a priori probabilities as equal to $m^{N}$, which is the number of ways of permuting $N$ particles among $m$ cells. Note also Bernoulli's case ${ }^{2}$ where the maximum of $P\left\{n_{i}\right\}$ is given by $n_{i}=N p$ for then $P\left\{n_{i}\right\} \approx 1$ when Stirling's approximation is made. The coincidence between the observed frequencies $n_{\mathrm{i}} / N$ and their expected values $p_{i}$ means that the fluctuations have disappeared, and this surely can occur only in the limiting case where $N$ and $m$ both tend to infinity such that their ratio is constant. The fact that $p_{1}^{n_{I}} \ldots p_{m}^{n_{m}}$ has been traded for the $m^{N}$ in his expression for the multinomial distribution is responsible for the absence of the degeneracy factor $m$ in his expressions (11) and (13) for Maxwell-Boltzmann statistics.

Tersoff and Bayer introduce unequal $a$ priori weighting factors into the multinomial expression but their result, the inverse negative binomial coefficient, disqualifies their results, as $p_{i}$ in their equation (3) does not conserve probability. There is nothing unique about their starting point as their multinomial 'distribution' is not a proper probability distribution. So their proposed 'distribution' has no closer connection to probability theory than the negative binomial coefficient itself, which is the traditional starting point for deriving Bose-Einstein statistics.

The crux of the matter lies rather in what physical situations are being considered. Take, for instance, the adsorption and de-adsorption of $N$ molecules onto a lattice of $m$ sites. Given that the probabilities per unit time for adsorption and deadsorption are proportional to $N$ and $m-$ $N$, respectively, the solution to the onestep master equation is the binomial distribution. The particles are indistinguishable, for it makes no difference which particle is adsorbed onto which lattice site. Alternatively, when one considers Einstein's ${ }^{4}$ derivation of Planck's radiation law, where emission and absorption of radi- 